\author{
United Nations Educational Scientific and Cultural Organization \\ and \\ International Atomic Energy Agency
}

THE ABDUS SALAM INTERNATIONAL CENTRE FOR THEORETICAL PHYSICS

\title{
PHASE DIAGRAMS OF A SPIN-1 ISING SUPERLATTICE WITH ALTERNATING TRANSVERSE FIELD
}

\author{
A. Saber \\ INFM, Dipartimento di Fisica, Università di Padova, \\ Via Marzolo 8, 35131 Padova, Italy, \\ Département de Physique, Faculté des Sciences, Université Moulay Ismail, \\ B.P. 4010, Meknès Morocco \\ and
}

The Abdus Salam International Centre for Theoretical Physics, Trieste, Italy,

H. Ez-Zahraouy ${ }^{1}$

Département de Physique, Laboratoire de Magnétisme et de Physique des Hautes Energies, Faculté des Sciences, B. P. 1014, Rabat, Morocco and

The Abdus Salam International Centre for Theoretical Physics, Trieste, Italy,

$$
\begin{gathered}
\text { G. Mattei } \\
\text { INFM, Dipartimento di Fisica, Università di Padova, } \\
\text { Via Marzolo 8, } 35131 \text { Padova, Italy }
\end{gathered}
$$

and

A. Ainane

INFM, Dipartimento di Fisica, Università di Padova,

Via Marzolo 8, 35131 Padova, Italy,

Département de Physique, Faculté des Sciences, Université Moulay Ismail, B.P. 4010, Meknès Morocco and

The Abdus Salam International Centre for Theoretical Physics, Trieste, Italy.

$$
\text { MIRAMARE - TRIESTE }
$$

September 2000

\footnotetext{
${ }^{1}$ Regular Associate of the Abdus Salam ICTP. E-mail: ezahamid@fsr.ac.ma
} 


\begin{abstract}
The effects of alternating transverse fields $\Omega_{a}$ and $\Omega_{b}$ on the critical behavior of an alternating spin-1 Ising superlattice are studied within an effective field theory with a probability distribution technique that accounts for the single-site spin correlations. Critical temperatures are calculated as a function of the thickness of the superlattice and the strength of the transverse field. Depending on the values of the transverse fields $\Omega_{a}$ and $\Omega_{b}$, the critical temperature can increase or decrease with increasing the thickness of the film, such result is not obtained in the uniform transverse field case $\left(\Omega_{a}=\Omega_{b}\right)$. Furthermore, for each thickness $L$ of the film, a long range ordered phase persists at low temperature for selected values of the transverse field $\Omega_{a}$ and arbitrary values of $\Omega_{b}$. The effects of interlayer and intralayer exchange interactions are also examined.
\end{abstract}




\section{INTRODUCTION}

During the last few years much effort has been directed towards the study of the critical phenomena in various magnetic layered structures and superlattices [1-7]. The basic theoretical problem is the examination of the magnetic excitation and the phase transitions in these systems. Magnetic excitations in superlattices were considered in numerous papers (see e.g. [8] for a breif review). Yet, less attention has been paid to the critical behavior and in particular to the critical temperatures in superlattices. Ma and Tasi [9] have studied the variation with modulation wavelength of the Curie temperature for a Heisenberg magnetic superlattice. Their results agree qualitatively with experiments on $C u / N i$ films [10]. Superlattice structures composed of alternating ferromagnetic and antiferromagnetic layers have been investigated by Hinckey and Mills [11,12], using a localized spin model. Magnetic properties of an alternating magnetic and nomagnetic superlattice is studied by Benyoussef and Ez-Zahraouy [13] using finite cluster approximatiom. A sequence of spin transitions is found to be different for superlattices with antiferromagnetic component consisting of an even or odd number of spin layers. In two earlier papers $[5,6]$ two of the present authors studied the effects of a uniform transverse field and surface magnetism on the critical behavior of an alternating ferromagnetic spin $-1 / 2$ Ising superlattice. Our aim in this paper is to study the effects of an alternating transverse field on the critical temperature of a spin-1 Ising superlattice consisting of two ferromagnetic materials with different bulk properties, with a simple cubic structure. In particular, we consider the two constituents $\mathrm{A}$ and $\mathrm{B}$ with different bulk transition temperatures, i.e. $T_{c}^{A} \neq T_{c}^{B}$. The interface is in general different in nature from both bulks, even if the bulk critical temperatures are the same. We use the effective field theory with a probability distribution technique in its simplest form [14-15]. This technique is believed to give more exact results than those of the standard mean-field approximation. In section 2 we outline the formalism and derive the equation that determines the transition temperature. Results and discussion are given in section 3. Section 4 is reserved for the onclusion.

\section{MODEL AND FORMULATION}

We consider an infinite simple cubic superlattice with a unit cell consisting of arbitrary number $L$ of magnetic layers. The Transverse spin-1 Ising Hamiltonian of the system is given by

$$
H=-\sum_{n, n^{\prime}} \sum_{r, r^{\prime}} J_{n n^{\prime}} \sigma_{n r}^{z} \sigma_{n^{\prime} r^{\prime}}^{z}-\sum_{n} \sum_{r} \Omega_{n} \sigma_{n r}^{x}
$$

where $\sigma_{n r}^{z}$ and $\sigma_{n r}^{x}$ denote respectively the $z$ and $x$ components of a quantum spin $\vec{\sigma}_{n r}$ of magnitude $\sigma_{n r}=1$ at site $(n, r),\left(n, n^{\prime}\right)$, are plane indices and $\left(r, r^{\prime}\right)$ are different sites of the

planes, and $J_{n n^{\prime}}$ is the strength of the ferromagnetic exchange interaction which is only plane dependent. We will retain only nearest-neighbor terms. For a fixed configuration of neighbouring 
spins of the site $(n, r)$ that the longitudinal and the transverse magnetizations and quadrupolar moments of any spin at site $(n, r)$ are given by,

$$
\begin{gathered}
m_{n r \alpha}=\left\langle\sigma_{n r}^{\alpha}\right\rangle=\left\langle f_{1 \alpha}(A, B)\right\rangle \\
q_{n r \alpha}=\left\langle\left(\sigma_{n r}^{\alpha}\right)^{2}\right\rangle=\left\langle f_{2 \alpha}(A, B)\right\rangle
\end{gathered}
$$

where $\alpha=z, x$ for the longitudinal and transverse magnetizations and quadrupolar moments respectively and

$$
\begin{gathered}
f_{1 z}(A, B)=\frac{A}{\left[A^{2}+B^{2}\right]^{1 / 2}} \frac{2 \sinh \left(\beta\left[A^{2}+B^{2}\right]^{1 / 2}\right)}{1+2 \cosh \left(\beta\left[A^{2}+B^{2}\right]^{1 / 2}\right)} \\
f_{2 z}(A, B)=\frac{1}{\left[A^{2}+B^{2}\right]} \frac{B^{2}+\left(2 A^{2}+B^{2}\right) \cosh \left(\beta\left[A^{2}+B^{2}\right]^{1 / 2}\right)}{1+2 \cosh \left(\beta\left[A^{2}+B^{2}\right]^{1 / 2}\right)}
\end{gathered}
$$

and

$$
\begin{aligned}
& f_{1 x}(A, B)=f_{1 z}(B, A) \\
& f_{2 x}(A, B)=f_{2 z}(B, A)
\end{aligned}
$$

with

$$
\begin{gathered}
A=\sum_{n^{\prime}} \sum_{r^{\prime}} J_{n n^{\prime}} \sigma_{n^{\prime} r^{\prime}}^{z}, \\
B=\Omega_{n}
\end{gathered}
$$

where $\beta=1 / k_{B} T,\langle\ldots\rangle$ indicates the usual canonical ensemble thermal average for a given configuration and the first and second sums run over all possible configurations of atoms environing or lying on the $(n, r)$ site, respectively. Each of these configurations can be characterized by numbers of magnetic atoms in the planes $n-1, n, n+1$. To perform thermal averaging on the right-hand side of equations (2) and (3) one now follows the general approach described in $[14,16]$. Thus with the use of the integral representation method of Dirac $\delta$-distribution, equations (2) and (3) can be written in the form

$$
\begin{gathered}
\left\langle\sigma_{n r}^{\alpha}\right\rangle=\int d \omega f_{1_{\alpha}}(\omega, B) \frac{1}{2 \pi} \int d t \exp (i \omega t) \prod_{n^{\prime} r^{\prime}}\left\langle\exp \left(-i t J_{n, n^{\prime}} \sigma_{n^{\prime} r^{\prime}}^{z}\right)\right\rangle \\
\left\langle\left(\sigma_{n r}^{\alpha}\right)^{2}\right\rangle=\int d \omega f_{2 \alpha}(\omega, B) \frac{1}{2 \pi} \int d t \exp (i \omega t) \prod_{n^{\prime} r^{\prime}}\left\langle\exp \left(-i t J J_{n, n^{\prime}} \sigma_{n^{\prime} r^{\prime}}^{z}\right)\right\rangle
\end{gathered}
$$

In the derivation of the equations (10) and (11), the commonly used approximation has been made according to which the multi-spin correlation functions are decoupled into products of the spin averages. Then, as $\left\langle\sigma_{n r}^{\alpha}\right\rangle$ and $\left\langle\left(\sigma_{n r}^{\alpha}\right)^{2}\right\rangle$ are independent of $r$, we introduce the longitudinal magnetization and the longitudinal quadrupolar moment of the $n-t h$ layer, on the basis of equations (2) and (3), with the use of the probability distribution of the spin variables $[13,14]$

$$
P\left(\sigma_{n r}^{z}\right)=\frac{1}{2}\left[\left(q_{n z}-m_{n z}\right) \delta\left(\sigma_{n r}^{z}+1\right)+2\left(1-q_{n z}\right) \delta\left(\sigma_{n r}^{z}\right)+\left(q_{n z}+m_{n z}\right) \delta\left(\sigma_{n r}^{z}-1\right)\right]
$$


Allowing for the site magnetizations and quadrupolar moments to take different values in each atomic layer parallel to the surfaces of the superlattice, and labeling them in accordance with the layer number in which they are situated, the application of Eqs. (2), (10) and (12) yields the following set of equations for the layer longitudinal magnetizations

$$
\begin{aligned}
m_{n \alpha}= & 2^{-N-2 N_{0}} \sum_{\mu=0}^{N} \sum_{\nu=0}^{N-\mu} \sum_{\mu_{1}=0}^{N_{0}} \sum_{\nu_{1}=0}^{N_{0}-\mu_{1}} \sum_{\mu_{2}=0}^{N_{0}} \sum_{\nu_{2}=0}^{N_{0}-\mu_{2}} 2^{\mu+\mu_{1}+\mu_{2}} C_{\mu}^{N} C_{\nu}^{N-\mu} \\
& C_{\mu_{1}}^{N_{0}} C_{\nu_{1}}^{N_{0}-\mu_{1}} C_{\mu_{2}}^{N_{0}} C_{\nu_{2}}^{N_{0}-\mu_{2}}\left(1-2 q_{n z}\right)^{\mu}\left(q_{n z}-m_{n z}\right)^{\nu}\left(q_{n z}+m_{n z}\right)^{N-\mu-\nu} \\
& \left(1-2 q_{n-1, z}\right)^{\mu_{1}}\left(q_{n-1, z}-m_{n-1, z}\right)^{\nu_{1}}\left(q_{n-1, z}+m_{n-1, z}\right)^{N_{0}-\mu_{1}-\nu_{1}} \\
& \left(1-2 q_{n+1, z}\right)^{\mu_{2}}\left(q_{n+1, z}-m_{n+1, z}\right)^{\nu_{2}}\left(q_{n+1, z}+m_{n+1, z}\right)^{N_{0}-\mu_{2}-\nu_{2}} f_{1 \alpha}\left(y_{n}, \Omega_{n}\right)
\end{aligned}
$$

where

$$
y_{n}=J\left[R(N-\mu-2 \nu)+\left(2 N_{0}-\mu_{1}-\mu_{2}-2 \nu_{1}-2 \nu_{2}\right)\right]
$$

$N$ and $N_{0}$ are the numbers of nearest neighbors in the plane and between adjacent planes respectively ( $N=4$ and $N_{0}=1$ in the case of a simple cubic lattice which is considered here) and $C_{k}^{l}$ are the binomial coefficients, $C_{k}^{l}=\frac{l !}{k !(l-k) !}$. The periodic conditions of the superlattice has to be satisfied, namely $m_{0 \alpha}=m_{L \alpha}, m_{L+1, \alpha}=m_{1 \alpha}$ and $q_{0 \alpha}=q_{L \alpha}$, and $q_{L+1, \alpha}=q_{1 \alpha}$. The equations of the longitudinal and transverse quadrupolar moments are obtained by substituting the function $f_{1 \alpha}$ by $f_{2 \alpha}$ in the expressions of the layer longitudinal and transverse magnetizations respectively. This yields

$$
q_{n \alpha}=m_{n \alpha}\left[f_{1 \alpha}\left(y_{n}, \Omega_{n}\right) \rightarrow f_{2 \alpha}\left(y_{n}, \Omega_{n}\right)\right]
$$

In this work we are interested with the calculation of the ordering near the transition critical temperature. The usual argument that $m_{n z}$ tends to zero as the temperature approaches its critical value, allows us to consider only terms linear in $m_{n z}$ because higher order terms tend to zero faster than $m_{n z}$ on approaching a critical temperature. Consequently, all terms of the order higher than linear terms in eqs. (13) that give the expressions of $m_{n z}$ can be neglected. This leads to the set of simultaneous equations

$$
m_{n z}=A_{n, n-1} m_{n-1, z}+A_{n, n} m_{n z}+A_{n, n+1} m_{n+1, z}
$$

or

$$
A \vec{m}_{z}=\vec{m}_{z}
$$

where $\vec{m}_{z}$ is a vector of components $\left(m_{1 z}, m_{2 z}, \ldots, m_{n z}, \ldots, m_{L z}\right)$ and the matrix $A$ is symmetric and tridiagonal with elements

$$
A_{i, j}=A_{i, i} \delta_{i, j}+A_{i, j}\left(\delta_{i, j-1}+\delta_{i, j+1}\right)
$$

The system of eqs. (17) is of the form

$$
M \vec{m}_{n z}=0
$$


where

$$
M_{i, j}=\left(A_{i, j}-1\right) \delta_{i, j}+A_{i, j}\left(\delta_{i, j-1}+\delta_{i, j+1}\right)
$$

The only non zero elements of the matrix $M$ are given by

$$
\begin{aligned}
M_{n, n-1}= & 2^{-N-2 N_{0}} \sum_{\mu=0}^{N} \sum_{\nu=0}^{N-\mu} \sum_{\mu_{1}=0}^{N_{0}} \sum_{\nu_{1}=0}^{N_{0}-\mu_{1}} \sum_{\mu_{2}=0}^{N_{0}} \sum_{\nu_{2}=0}^{N_{0}-\mu_{2}} \sum_{i=0}^{\nu_{1}} \sum_{j=0}^{N_{0}-\left(\mu_{1}+\nu_{1}\right)}(-1)^{i} 2^{\mu+\mu_{1}+\mu_{2}} \delta_{1, i+j} \\
& C_{\mu}^{N} C_{\nu}^{N-\mu} C_{\mu_{1}}^{N_{0}} C_{\nu_{1}}^{N_{0}-\mu_{1}} C_{\mu_{2}}^{N_{0}} C_{\nu_{2}}^{N_{0}-\mu_{2}} C_{i}^{\nu_{1}} C_{j}^{N_{0}-\left(\mu_{1}+\nu_{1}\right)}\left(1-r_{n}\right)^{\mu} \\
& \left(1-r_{n-1}\right)^{\mu_{1}}\left(1-r_{n+1}\right)^{\mu_{2}} r_{n}^{N-\mu_{2}} r_{n-1}^{\left(N_{0}-\mu_{1}\right)-(i+j)} r_{n+1}^{N_{0}-\mu_{2}} f_{1 z}\left(y_{n}, \Omega_{n}\right) \\
M_{n, n}= & 2^{-N-2 N_{0}} \sum_{\mu=0}^{N} \sum_{\nu=0}^{N-\mu} \sum_{\mu_{1}=0}^{N_{0}} \sum_{\nu_{1}=0}^{N_{0}-\mu_{1}} \sum_{\mu_{2}=0}^{N_{0}} \sum_{\nu_{2}=0}^{N_{0}-\mu_{2}} \sum_{i=0}^{\nu} \sum_{j=0}^{N_{-}(\mu+\nu)}(-1)^{i} 2^{\mu+\mu_{1}+\mu_{2}} \delta_{1, i+j} \\
& C_{\mu}^{N} C_{\nu}^{N-\mu} C_{\mu_{1}}^{N_{0}} C_{\nu_{1}}^{N_{0}-\mu_{1}} C_{\mu_{2}}^{N_{0}} C_{\nu_{2}}^{N_{0}-\mu_{2}} C_{i}^{\nu} C_{j}^{N-(\mu+\nu)}\left(1-r_{n}\right)^{\mu} \\
& \left(1-r_{n-1}\right)^{\mu_{1}}\left(1-r_{n+1}\right)^{\mu_{2}} r_{n}^{N-\mu-(i+j)} r_{n-1}^{\left(N_{0}-\mu_{1}\right)} r_{n+1}^{N_{0}-\mu_{2}} f_{1 z}\left(y_{n}, \Omega_{n}\right)-1 \\
M_{n, n+1}= & 2^{-N-2 N_{0}} \sum_{\mu=0}^{N} \sum_{\nu=0}^{N-\mu} \sum_{\mu_{1}=0}^{N_{0}} \sum_{\nu_{1}=0}^{N_{0}-\mu_{1}} \sum_{\mu_{2}=0}^{N_{0}} \sum_{\nu_{2}=0}^{N_{0}-\mu_{2}} \sum_{i=0}^{\nu_{2}} \sum_{j=0}^{N_{0}-\left(\mu_{2}+\nu 2\right)}(-1)^{i} 2^{\mu+\mu_{1}+\mu_{2}} \delta_{1, i+j} \\
& C_{\mu}^{N} C_{\nu}^{N-\mu} C_{\mu_{1}}^{N_{0}} C_{\nu_{1}}^{N_{0}-\mu_{1}} C_{\mu_{2}}^{N_{0}} C_{\nu_{2}}^{N_{0}-\mu_{2}} C_{i}^{\nu_{1}} C_{j}^{N_{0}-\left(\mu_{2}+\nu 2\right)}\left(1-r_{n}\right)^{\mu} \\
& \left(1-r_{n-1}\right)^{\mu_{1}}\left(1-r_{n+1}\right)^{\mu_{2}} r_{n}^{N-\mu_{1}} r_{n-1}^{\left(N_{0}-\mu_{1}\right)} r_{n+1}^{N_{0}-\mu_{2}-(i+j)} f_{1 z}\left(y_{n}, \Omega_{n}\right)
\end{aligned}
$$

where

$$
\begin{aligned}
r_{n}= & 2^{-N-2 N_{0}} \sum_{\mu=0}^{N} \sum_{\nu=0}^{N-\mu} \sum_{\mu_{1}=0}^{N_{0}} \sum_{\nu_{1}=0}^{N_{0}-\mu_{1}} \sum_{\mu_{2}=0}^{N_{0}} \sum_{\nu_{2}=0}^{N_{0}-\mu_{2}} 2^{\mu+\mu_{1}+\mu_{2}} C_{\mu}^{N} C_{\nu}^{N-\mu} \\
& C_{\mu_{1}}^{N_{0}} C_{\nu_{1}}^{N_{0}-\mu_{1}} C_{\mu_{2}}^{N_{0}} C_{\nu_{2}}^{N_{0}-\mu_{2}}\left(1-2 r_{n}\right)^{\mu} r_{n}^{N-\mu}\left(1-r_{n-1}\right)^{\mu_{1}} r_{n-1}^{\left(N_{0}-\mu_{1}\right)} \\
& \left(1-2 r_{n+1}\right)^{\mu_{2}} r_{n+1}^{N_{0}-\mu_{2}} f_{2 z}\left(y_{n}, \Omega_{n}\right)
\end{aligned}
$$

All the information about the critical temperature of the system is contained in eq. (19). Up to now we did not precise the values of the exchange interactions; the terms in matrix (19) are general ones. In a general case, for arbitrary coupling constants and superlattice thickness the evaluation of the critical temperature relies on the numerical solution of the system of linear equations (19). These equations are fulfilled if and only if

$$
\operatorname{det} M=0
$$

This condition can be satisfied for $L$ different values of the critical temperature $T_{c}$. Hereafter, we will take $J_{a a}$ as the unit of the energy, the length is measured in units of the lattice constant and we introduce the reduced exchange couplings $R_{1}=J_{b b} / J_{a a}$ and $R_{2}=J_{a b} / J_{a a}$. Let us begin with the evaluation of the critical temperature with an example: the critical temperature of the 
spin-1 Ising model for the simplest possible "bulk case" of a material $A$ (i.e. $N=4, N_{0}=1$, $J_{i, j}=J_{a a}, \Omega_{n}=\Omega$ ). Then we can reduce $\operatorname{det} M$ to the following form

$$
\operatorname{det} M=\left|\begin{array}{cccccccccccc}
a & b & & & & & & & & & & b \\
b & a & b & & & & & & & & & \\
& b & a & b & & & & & & & & \\
\ldots & \ldots & \ldots & \ldots & \ldots & \ldots & \ldots & \ldots & \ldots & \ldots & \ldots & \ldots \\
& & & & b & a & b & & & & & \\
\cdots & \ldots & \ldots & \ldots & \ldots & \ldots & \ldots & \ldots & \ldots & \ldots & \ldots & \ldots \\
& & & & & & & b & a & b & & \\
& & & & & & & & b & a & b & \\
b & & & & & & & & & & b & a
\end{array}\right|_{(L, L)}
$$

whose values are

$$
\operatorname{det} M_{b u l k}=\prod_{k=1}^{L}\left[a+2 b \cos \left(\frac{2 \pi(k-1)}{L}\right)\right]
$$

where the elements in the above determinant are given by

$$
\begin{gathered}
a=M_{n, n}\left(J_{n, n}=J_{n, n-1}=J_{n, n+1}=J_{a a}, \Omega\right) \\
b=\frac{1}{4}(a+1)
\end{gathered}
$$

and $L$ in the "bulk" case is an arbitrary number. Now we obtain the critical temperature from the condition given by

$$
\operatorname{det} M_{b u l k}=0
$$

We apply the obtained formalism to an alternating magnetic superlattice consisting of atoms of type $A$ and $B$ which alternate as ...ABABAB...AB...in alternating transverse magnetic fields $\Omega_{a}, \Omega_{b}$ respectively. The periodic conditions suggest that we only have to consider one unit cell which interacts with its nearest neighbors via the interlayer coupling. The coupling strength between nearest-neighboring spins in A and B is denoted by $J_{a a}$ and $J_{b b}$ respectively, while $J_{a b}$ stands for the exchange coupling between the nearest-neighbor spins for all successive layers. Let us consider a simple alternating lattice of $2 L$ layers $n=1,3,5 \ldots 2 L-1$ consist of atoms of type A, whereas layers $n=2,4, \ldots 2 L$ consist of atoms of type B. In this case we can represent $\operatorname{det} M_{a b}$ as

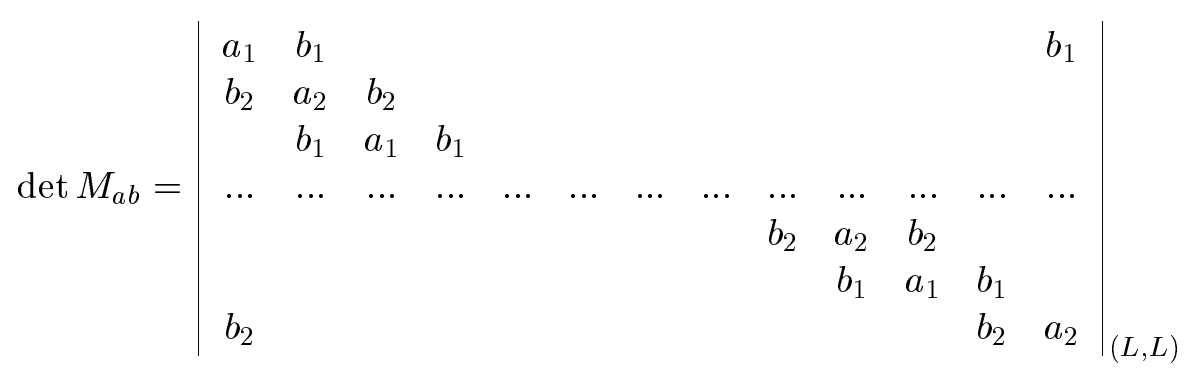

whose value is

$$
\operatorname{det} M_{a b}=\left(a_{1} a_{2}\right)^{L} \prod_{k=1}^{L}\left\{1-\frac{2 b_{1} b_{2}}{a_{1} a_{2}}\left[1+\cos \left(\frac{2 \pi(k-1)}{L}\right)\right]\right\}
$$


where the elements in the determinant are given by

$$
\begin{gathered}
\left\{\begin{array}{c}
a_{1}=M_{n, n}\left(J_{n, n}=J_{a a}, J_{n, n-1}=J_{n, n+1}=J_{a b}, \Omega_{a}\right) \\
b_{1}=M_{n, n-1}\left(J_{n, n}=J_{a a}, J_{n, n-1}=J_{n, n+1}=J_{a b}, \Omega_{a}\right) n=1,3, \ldots 2 L-1 \\
=M_{n, n+1}\left(J_{n, n}=J_{a a}, J_{n, n-1}=J_{n, n+1}=J_{a b}, \Omega_{a}\right)
\end{array}\right. \\
\left\{\begin{array}{c}
a_{2}=M_{n, n}\left(J_{n, n}=J_{b b}, J_{n, n-1}=J_{n, n+1}=J_{a b}, \Omega_{b}\right) \\
b_{2}=M_{n, n-1}\left(J_{n, n}=J_{b b}, J_{n, n-1}=J_{n, n+1}=J_{a b}, \Omega_{b}\right) n=2,4, \ldots 2 L \\
=M_{n, n+1}\left(J_{n, n}=J_{b b}, J_{n, n-1}=J_{n, n+1}=J_{a b}, \Omega_{b}\right)
\end{array}\right.
\end{gathered}
$$

$L$ is the thickness of the superlattice, it is an arbitrary number. Now we obtain the critical temperature of the system from the condition given by

$$
\operatorname{det} M_{a b}=0 \text {. }
$$

\section{Results and discussion}

From the numerical solution of eq. (35), the critical temperature of the alternating superlattice is calculated as a function of the reduced exchange interactions $R_{1}$ and $R_{2}$. We assume that $R_{1} \leq 1$ and denote by $T_{c}^{a} / J_{a a}=3.5186$ and by $T_{c}^{b} / J_{a a}=\left(T_{c}^{a} / J_{a a}\right) R_{1}$ the bulk critical temperature of a uniform lattice of material $\mathrm{A}$ and of material $\mathrm{B}$. In the case of a uniform transverse field $\left(\Omega_{a}=\Omega_{b}\right)$, Fig.1a shows the dependence of the critical temperature $T_{c} / J_{a a}$ on the reduced interlayer exchange coupling $R_{2}$ for various values of the transverse field and $R_{1}=1$. This dependence is quasi linear, in good agreement with the results obtained in the $\mathrm{spin}-1 / 2$ case [17-18]. From Fig.1b, it is clear that the critical temperature increases with increasing the thickness of the superlattice. Besides this we note that the critical temperature increases with increasing $R_{2}$ and/or decreasing the value of the transverse field $\Omega$.

Now, in order to examine the effects of the alternating transverse fields $\Omega_{a}$ and $\Omega_{b}$, we will neglect the effect of the competition between interlayer and intralayer exchange interactions, for this reason it is practical to choose $R_{1}=R_{2}=1$. We note that there is a symmetry between $\Omega_{a}$ and $\Omega_{b}$ (i.e if we change $\Omega_{a}$ by $\Omega_{b}$ and vice versa the results remain the same). The results presented in Fig.2 show the effects of the alternating transverse field on the critical temperature $T_{c} / J_{a a}$ of the superlattice with a fixed thickness $L(L=10)$. However, $T_{c}$ decreases with increasing $\Omega_{b}$. Furthermore, there exists a critical transverse field $\Omega_{a}^{c}$, below which the system remains ordered at low temperature for any value of $\Omega_{b}$ (this is called the long range ordered phase), while for $\Omega_{a}$ sufficiently large the system exhibits an ordered-disordered phase transition at very low temperture (in particular at $T=0$ ) and the long range order disappears. Such behavior does not occur for very large thickness $L$ and weak strength transverse field $\Omega_{a}$ (Fig.3a), while for $\Omega_{a}$ sufficiently large, and for $L$ large $(L=32)$ the long range ordered phase occurs at low temperature (Fig.3b). These results mean that the critical transverse field $\Omega_{a}^{c}$ mentioned above depends on the thickness value of the superlattice. Depending on the values of the alternating transverse fields, the critical temperature undergoes two different behaviors as a 
function of the thickness of the superlattice. Indeed from Fig. 3a it is clear that $T_{c} / J_{a a}$ decreases with increasing the thickness $L$ of the superlattice for sufficiently weak values of $\Omega_{a}$ and large value of $\Omega_{b}$, while it increases with increasing the thickness $L$ for $\Omega_{a}$ large and small value of $\Omega_{b}$. Such results are not obtained in the uniform transverse field case $\left(\Omega_{a}=\Omega_{b}\right)$. We denote that the critical temperature vanishes at the same critical transverse fields $\Omega_{b}$ for any thickness $L$, which is different than the bulk one (Fig.3b). In Fig. 4 we give the behavior of $T_{c}$ as a function of the thickness of the superlattice from which it is clear that $T_{c}$ decreases with increasing the thickness $L$ for weak enough $\Omega_{a}$ and large values of $\Omega_{b}$ while it increases with $L$ in the contrary case.

\section{Conclusion}

We have investigated the effects of alternating transverse fields on the critical behavior of a spin-1 Ising superlattice formed by two alternating ferromagnetic materials using an effective field theory. On one hand it is found that the ordered phase persists at low temperature for sufficiently weak value of $\left(\Omega_{a} / J_{a a}\right)$, and arbitrary value of $\Omega_{b} / J_{a a}$, such behavior is not obtained in the uniform transverse field case. On the other hand, depending on the values of the transverse field $\Omega_{a}$ and $\Omega_{b}$, the critical temperature can increase or decrease with increasing the thickness of the superlattice.

\section{Acknowledgments}

The authors A.S., H. Ez and A.A. would like to thank UNESCO, IAEA and the Abdus SalamInternational Centre for Theoretical Physics, Trieste, Italy, for their hospitality. The authors A.S. and A.A. thank the "AS-ICTP TRIL Program" for the financial support and the collaboration between Padova University and CNCPRSPT Morocco. The author H. Ez would like also to thank the Arab Foundation for the Scholarship Association at the AS-ICTP, Italy. This work was done within the framework of the Associateship Scheme of the Abdus Salam ICTP. 


\section{References}

[1] R. E. Cameley and R. L. Stamps, J. Phys.: Condens Matter 5, 3727 (1993)

[2] G. Shaulov and Yu. Seidov, J. Magnetism Magn. Materr. 140/144, 527 (1995)

[3] T. Balcerzak and J. W. Tucker, J. Magnetism Magn. Mater. 140/144, 653 (1995)

[4] T. Hai, Z. V. Li, D. L. Lin, and Th. F. George, J. Magnetism Magn. Mater. 97, 227 (1991)

[5] I. Essaoudi, A. Saber, A. Ainane, M. Saber, Phys. Scr. 54 (1999) 168-173

[6] A. Saber, I. Essaoudi, A. Ainane, and M. Saber, Phys. Status Solidi (b) 209, 161 (1998)

[7] A. Saber, A. Ainane, M. Saber, I. Essaoudi, F. Dujardin, and B. Stébé, Phys. Rev. B. 60, 4149 (1999)

[8] J. A. Morkowski and A. Szajek J. Magnetism Magn. Mater. 71, 299 (1988)

[9] H. R. Ma and C. H. Tasi, Solid State Commun; 55, 499 (1985)

[10] J. Q. Zheng, J. B. Keterson, C. M. Falco, and I. K. Schuller, J. Appl. Phys. 53, 3150 (1982)

[11] L. L. Hinchey and D. L. Mills, Phys. Rev. B 33, 3329 (1986)

[12] L. L. Hinchey and D. L. Mills, Phys. Rev. B 34, 1689 (1986)

[13] Benyoussef A, Ez-Zahraouy H, Phys. Rev. B 52, 4245 (1995)

[14] Elkouraychi A, Saber M and Tucker J. W. 1995 Physica A 213, 576

[15] Saber M 1987 J. Phys. C: Solid State Phys. 20, 2749

[16] Kanyoshi: T 1991 J. Phys.: Condens. Matter 34497

[17] Sy, H. K. and Ow, M. H., J. Phys.: Condens. Matter 4, 5891 (1992)

[18] Y. M. Seidov and G. R. Shaulov, J. Phys.: Condensed Matter 6, 9621 (1994) 


\section{Figure Captions}

Fig 1. Critical temperature $T_{c} / J_{a a}$ as a function of $R_{2}($ a) and the tickness of the superlattice (b). In (b) the number accompanying each curve denotes the value of $R_{2}$, while the solid and dashed lines correspond to $\Omega / J_{a a}=0,1$ respectively.

Fig 2. The dependence of the critical temperature $T_{c} / J_{a a}$ on the transverse field $\Omega_{b} / J_{a a}$ for $L=10$.

Fig 3. The dependence of the critical temperature $T_{c} / J_{a a}$ as a function of the transverse field $\Omega_{b}$ for (a) $\Omega_{a} / J_{a a}=0.5$ and (b) $\Omega_{a} / J_{a a}=2$.

Fig 4. The dependence of the critical temperature $T_{c} / J_{a a}$ on the thickness $L$ of the finite superlattice. 